\title{
Spectrum construction of differentially expressed circular RNAs in patients with leukoaraiosis and function analysis of differentially expressed genes
}

\author{
TE MI ${ }^{1}$, CHANGJIANG LUO ${ }^{2}$, YAWEI HU ${ }^{3}$, CHUANQIANG QU ${ }^{4}$, \\ XIANG WANG ${ }^{4}$, SHOUGANG GUO $^{4}$ and YIFENG DU ${ }^{4}$
}

${ }^{1}$ Department of ICU, Jining No. 1 People's Hospital, Jining, Shandong $272000 ;{ }^{2}$ Department of Neurology, Provincial Hospital Affiliated to Shandong University (South Branch), Jinan, Shandong 250002; ${ }^{3}$ Department of Neurosurgery, Affiliated Hospital of Jining Medical University Jining, Shandong 272029; ${ }^{4}$ Department of Neurology, Provincial Hospital Affiliated to Shandong University, Jinan, Shandong 250021, P.R. China

Received March 26, 2016; Accepted March 3, 2017

DOI: $10.3892 / \mathrm{mmr} .2017 .6871$

\begin{abstract}
Circular RNAs (circRNAs) are class of endogenous RNAs that have a role in the regulation of gene expression. The present study aimed to investigate the diagnostic value and role of circRNA in the pathogenesis of leukoaraiosis (LA). The present study performed Arraystar Human circRNA Array analysis of 6 samples from LA cases and 6 samples from control cases. Differentially expressed (DE) circRNAs between two samples were identified through fold-change (>1.5-fold) screening. Afterwards, based on DE circRNAs, the gene ontology (GO) analysis of upregulated DE genes identified from DE circRNAs demonstrated that DE genes were primarily associated with cellular metabolic processes, membrane-bound organelles and binding. However, none were enriched in the Kyoto Encyclopedia of Genes and Genomes (KEGG) pathway. Downregulated DE genes were enriched in cellular localization, cytoplasm and kinase binding. For the KEGG pathways, the downregulated DE genes were primarily associated with the insulin signaling pathway. The results of the present study indicated that the DE genes from differently expressed circRNAs may have an important role in the pathogenesis of LA and may be a novel targfet for further research.
\end{abstract}

\section{Introduction}

Leukoaraiosis (LA), a common form of cerebral white matter lesion (WML), is characterized by punctuate or patchy

Correspondence to: $\mathrm{Dr}$ Chuanqiang $\mathrm{Qu}$, Department of Neurology, Provincial Hospital Affiliated to Shandong University, 324 Jingwuweiqi Road, Jinan, Shandong 250021, P.R. China

E-mail:drqucq@sina.com

Key words: circular RNAs, leukoaraiosis, biomarker, differentially expressed gene, gene ontology, Kyoto Encyclopedia of Genes and Genomes hyperintensities in the periventricular or subcortical white matter observed via magnetic resonance imaging (MRI). Although it remains asymptomatic, LA is not considered to be benign, and has been demonstrated to be associated with poor clinical outcomes and increased risk of disability, dementia, depression, stroke, and the overall morbidity and mortality (1). LA is associated with increased age, hypertension, diabetes mellitus, history of stroke and chronic atherosclerotic diseases (2). As hypertension is reported to be one of the major determinants of WMLs, the present study only included subjects with a history of hypertension. Despite intensive research, an understanding of the pathological mechanism remains incomplete, and is likely to be multifactorial. Therefore, identifying biomarkers is essential to improve the early diagnosis of LA.

Circular RNAs (circRNAs) are a class of endogenous RNAs that have a stable structure (3), which is a clear advantage of using circRNAs as a diagnostic marker. Previous studies have demonstrated that circRNAs are involved in the development of several types of diseases, including nervous system disorders (4,5). However, limited information is available regarding the association of circRNAs with LA. The present study, based on the hypertension population, compared the circRNA expression profiles of LA sufferers and controls. By performing a circRNA array analysis and reverse transcription-quantitative polymerase chain reaction (RT-qPCR), the present study demonstrated that certain circRNAs, including has_circ_102533 and has_circ_103783, may have potential as a novel type of biomarker for the diagnosis of LA. In addition, the gene ontology and KEGG analysis of differentially expressed (DE) genes may contribute to an improved understanding of the pathogenesis of LA.

\section{Materials and methods}

Subjects and clinical specimens. The current study was performed in accordance with the guidelines of the Helsinki Declaration. Written informed consent was obtained from all subjects and the Ethics Committee of Shandong Provincial 
Hospital Affiliated to Shandong University (Jinan, China) approved all aspects of the present study. A total of 30 subjects were recruited between August 2014 and September 2015 at Shandong Provincial Hospital Affiliated to Shandong University. All cases had a history of hypertension for 5-15 years. The groups were as follows: Test (LA sufferers; $\mathrm{n}=20 ; 7$ males and 13 females) and control (no LA; $\mathrm{n}=10$; 3 males and 7 females). The mean age was $58 \pm 5.7$ years. The subjects had a mean systolic pressure of $150 \pm 7.1 \mathrm{mmHg}$ and a mean diastolic pressure of $84 \pm 4.2 \mathrm{mmHg}$. LA is defined as punctuate or patchy hyperintensities in the periventricular or subcortical white matter, observed by MRI. Hypertension is defined as a history of high blood pressure $(\geq 140 / 90 \mathrm{mmHg})$ reported by the respondent, or by the current use of antihypertensive medication. None of the cases suffered from diabetes mellitus, heart diseases, dyslipidemia, hypotension, immune system diseases, hematological disorders, malnutrition, malignant tumors, serious liver and kidney diseases (including severe hepatitis or nephritis), liver or kidney failure, or had a history of heavy smoking (20 cigarettes daily) or alcoholism. Fresh fasting peripheral blood samples $(3 \mathrm{ml})$ were collected early in the morning and subsequently stored at $-80^{\circ} \mathrm{C}$.

Microarray expression analysis. Based on random sampling, Arraystar Human circRNA Microarray analysis version 2.0 (Arraystar, Inc., Rockville, MD, USA) of 6 samples from LA cases and 6 samples from control cases was performed. The sample preparation and microarray hybridization were performed based on the Arraystar's standard protocols. Total RNA from each sample was quantified using the NanoDrop ${ }^{\circledR}$ ND-1000 (NanoDrop Technologies; Thermo Fisher Scientific, Inc., Wilmington, DE, USA). RNA Integrity and genomic DNA contamination test was performed by denaturing agarose gel electrophoresis. Sample labeling and array hybridization were performed according to the manufacturer's protocol (Arraystar Inc.). Briefly, 2,600 ng/ $\mu 1$ total RNA (including 28s and 18s ribosomal RNA from each sample was treated with RNase R (Epicentre, Inc.) to enrich circRNA. The enriched RNA was subsequently amplified and transcribed into fluorescent complementary RNA (cRNA) utilizing random primers according to the Arraystar Super RNA Labeling kit protocol (Arraystar, Inc.). The labeled cRNAs were hybridized onto the Arraystar Human circRNA Mircoarrays (8x15K; Arraystar, Inc.) and incubated for $17 \mathrm{~h}$ at $65^{\circ} \mathrm{C}$ in an Agilent hybridization oven. After washing four times in hybridization buffer the labeled cRNAs, slides were scanned with an Agilent G2505C Microarray Scanner System (Agilent Technologies, Inc., Santa Clara, CA, USA).

Data collection and analysis. Scanned images were imported into Agilent Feature Extraction software version 11.0.1.1 (Agilent Technologies, Inc.) for raw data extraction. Quantile normalization of raw data and subsequent data processing were performed using the R software package (R Project for Statistical Computing, Vienna, Austria). After quantile normalization of the raw data, low intensity filtering was performed, and the circRNAs that were flagged as ' $\mathrm{P}$ ' or ' $\mathrm{M}$ ' ('All Targets Value') in at least 3 out of 6 samples were retained for further analyses. When comparing profile differences between two groups (including disease vs. control), the 'fold change' (i.e., the ratio of the group mean averages) between the groups for each circRNA was computed. The statistical significance of the difference was determined by a t-test. circRNAs with fold changes $>1.5$ and $\mathrm{P}<0.05$ were selected as significantly differentially expressed circRNAs. circRNA sequences were predicted by bioinformatics methods, as described previously (6).

RT-qPCR detection of hsa_circ_102470, hsa_circ_101396, hsa_circ_102533 and hsa_circ_103783. Total RNA in plasma was extracted using TRIzol $^{\oplus}$ reagent (Invitrogen; Thermo Fisher Scientific, Inc., Waltham, MA, USA) and was quantified using the NanoDrop ${ }^{\circledR}$ ND-1000, according to the manufacturer's protocol. cDNA was synthesized by RT (Arraystar Super RNA Labeling kit; Arraystar, Inc., Rockville, MD, USA) 3 times using random primers and the Gene Amp PCR system 9700, according to the manufacturer's protocol. A total of $1 \mu \mathrm{g}$ RNA, $1 \mu 1$ random (N9), $1.6 \mu 1$ dNTP mix (2.5 mM dATP, dGTP, dCTP, and dTTP, provided by HyTest Ltd) was combined to make the annealing mixture. This mixture was incubated in a $65^{\circ} \mathrm{C}$ water bath for $5 \mathrm{~min}$ and then put on ice for $2 \mathrm{~min}$. After brief centrifugation at $12,000 \mathrm{x}$ g or $15 \mathrm{~min}$ at $4^{\circ} \mathrm{C}$, the RT reactant solution $(4 \mu \mathrm{l} 5 \mathrm{X}$ First-Strand Buffer, $1 \mu \mathrm{l} 0.1 \mathrm{M}$ DTT, $0.3 \mu \mathrm{l}$ RNase inhibitor and $0.2 \mu \mathrm{l}$ SuperScript III RT, provided by Invitrogen; Thermo Fisher Scientific, Inc.) was added to a centrifuge tube and incubated at $37^{\circ} \mathrm{C}$ in a water bath for $1 \mathrm{~min}$. The tube was then incubated at $50^{\circ} \mathrm{C}$ in a water bath for $60 \mathrm{~min}$, followed by $70^{\circ} \mathrm{C}$ for $15 \mathrm{~min}$. Subsequently, cDNA was placed on ice. qPCR was performed using Arraystar $\mathrm{SYBR}^{\circledR}$-Green qPCR Master Mix (ROX-), $5 \mathrm{ml}$ on the $\mathrm{ViiA}^{\mathrm{TM}}$ 7 Real-time PCR system. Cycling conditions were as follows: An initial predenaturation step at $94^{\circ} \mathrm{C}$ for $20 \mathrm{sec}$, followed by 40 cycles of denaturation at $95^{\circ} \mathrm{C}$ for $10 \mathrm{sec}$, annealing at $60^{\circ} \mathrm{C}$ for $60 \mathrm{sec}$ and extension at $72^{\circ} \mathrm{C}$ for $15 \mathrm{sec}$. The primers used to quantify levels of the 4 circRNAs (hsa_circ_102470, hsa_circ_101396, hsa_circ_102533 and hsa_circ_103783) and GAPDH are presented in Table I. The data were analyzed using the relative values following internal calibration, presented as hsa_circ_102470/GAPDH, hsa_circ_101396/GAPDH, hsa_circ_102533/GAPDH and hsa_circ_103783/GAPDH. Subsequently, the ratios (average relative values in test group divided by the average relative values in control group) of the 4 circRNAs were further analyzed. The $2^{-\Delta \Delta C q}$ method was used for quantification (5).

Prediction of $D E$ genes and the function analysis of $D E$ genes. GO covers three domains, including biological process, cellular component and molecular function. Fisher's exact test is used by professionals to establish whether there is a higher overlap between the DE list and the GO annotation list than expected by chance. The P-value denotes the significance of GO term enrichment in the DE genes. The lower the P-value, the higher the significance of the GO Term $(\mathrm{P}<0.05$ is recommended). Pathway analysis is a functional analysis that maps genes to KEGG pathways. The P-value (EASE-score, Fisher-P-value or Hypergeometric-P-value) denotes the significance of the pathway associated with the conditions; the lower the P-value, the higher the significance of the pathway (The recommended P-value cut-off is 0.05 ). 
Table I. Primers for reverse transcription-quantitative polymerase chain reaction.

\begin{tabular}{|c|c|c|c|c|}
\hline \multirow[b]{2}{*}{ Gene } & \multicolumn{2}{|c|}{ Sequence } & \multirow{2}{*}{$\begin{array}{l}\text { Annealing } \\
\text { temperature }\left({ }^{\circ} \mathrm{C}\right)\end{array}$} & \multirow{2}{*}{$\begin{array}{l}\text { Product } \\
\text { length (bp) }\end{array}$} \\
\hline & Forward & Reverse & & \\
\hline GAPDH & 5'GGGAAACTGTGGCGTGAT3' & 5'GAGTGGGTGTCGCTGTTGA3' & 60 & 299 \\
\hline $\begin{array}{l}\text { hsa } \\
\text { circRNA_ } \\
102533\end{array}$ & 5'GCTGCCAAAAGCATAACCAA3' & 5'CCССТTTTCTGCTAAATGAACTCT3' & 60 & 198 \\
\hline $\begin{array}{l}\text { hsa } \\
\text { circRNA_ } \\
103783\end{array}$ & 5'AAGCTGTTAGCATGATCCCACC3' & 5'GATGAAACTTTTCCAAGTGTGGC3' & 60 & 133 \\
\hline $\begin{array}{l}\text { hsa_ } \\
\text { circRNA_ } \\
101396\end{array}$ & 5'AAAGGTCCACTTCGTATGCTG3' & 5'ACTCTGTCATTGGAGCAACTGTAT3' & 60 & 221 \\
\hline $\begin{array}{l}\text { hsa } \\
\text { circRNA_ } \\
102470\end{array}$ & 5'CCTAAATTTCACGACACCAG3' & 5'ATTCAGATTGCTCAAGGTAACT3' & 60 & 144 \\
\hline
\end{tabular}

circRNA, circular RNA.

Table II. Subject characteristics.

\begin{tabular}{|c|c|c|c|c|}
\hline Characteristic & Total & LA & Control & P-value \\
\hline Age, years & $58 \pm 5.7$ & $59 \pm 6.3$ & $56 \pm 2.4$ & 0.074 \\
\hline Number of males (\%) & $10(33.3)$ & $7(35)$ & $3(30)$ & 0.784 \\
\hline Systolic pressure, $\mathrm{mmHg}$ & $150( \pm 7.1)$ & $148( \pm 3.2)$ & $153( \pm 11.0)$ & 0.059 \\
\hline Diastolic pressure, $\mathrm{mmHg}$ & $84( \pm 4.2)$ & $82( \pm 3.9)$ & $85( \pm 4.6)$ & 0.162 \\
\hline
\end{tabular}

Data are presented as the mean \pm standard deviation (continuous variables) or as a proportion (discrete variables). LA, leukoaraiosis.

Statistical analysis. Data are presented as the mean \pm standard deviation (continuous variables) or as a proportion (discrete variables). The differences between groups were analyzed by Student's t-test (continuous variables) or chi-squared test (discrete variables). SPSS 17.0 software (SPSS, Inc., Chicago, IL, USA) was used for statistical analysis.

\section{Results}

Differential expression of circRNAs. The characteristics of age- and sex-matched test and control group subjects are presented in Table II. Compared with the controls, certain circRNAs exhibited $>1.5$-fold change $(\mathrm{P}<0.05)$ in the LA group; 32 were upregulated and 132 were downregulated. Among the upregulated circRNAs, hsa_circ_103783, hsa_circ_101396 and hsa_circ_102533 exhibited the highest statistically significant difference in expression, while of the downregulated circRNAs, hsa_circ_102470 was identified to exhibit the largest difference in expression between the LA and control groups. The results of the RT-qPCR detection of hsa_circ_102470, hsa_circ_101396, hsa_circ_102533 and hsa_circ_103783 are presented in Fig. 1. The expression of hsa_circ_103783 and hsa_circ_102533 in the test group was significantly higher compared with the control group, with fold increases in expression of $3.67(\mathrm{P}<0.001)$ and 3.52 $(\mathrm{P}<0.05)$, respectively. The expression of hsa_circ_101396 was 1.25 -fold higher in the test group compared with the control; however, this was not statistically significant $(\mathrm{P}=0.05)$. Comparing the expression of hsa_circ_102470 between the two groups, a 0.8 -fold downregulation, which was not statistically significant, was observed in the test group $(\mathrm{P}=0.39)$.

GO analysis of DE genes. The top 10 most significant enrichment GO terms of the upregulated DE genes are presented in Tables III-V. The biological process was primarily enriched in metabolic processes, including nucleobase-containing compounds, and heterocycle and cellular aromatic compound metabolic processes. The major cellular components were organelles, membrane-bounded organelles, intracellular membrane-bounded organelles and intracellular organelles. The molecular function predominantly enriched in the activity of histone acetyltransferase and acetyl-CoA were L-lysine, N6-acetyltransferase and ion channel activity. The top 10 most significantly enriched GO terms among the downregulated DE genes are presented in Tables VI-VIII. The present study 
Table III. Top ten gene ontology BP terms that upregulated genes were enriched in.

\begin{tabular}{lcc}
\hline & $\begin{array}{c}\text { Enrichment } \\
\text { BP term, metabolic process }\end{array}$ & P-value \\
\hline Nucleobase-containing compound & 2.66 & 0.002 \\
Heterocycle & 2.51 & 0.003 \\
Cellular aromatic compound & 2.50 & 0.003 \\
Organic cyclic compound & 2.33 & 0.004 \\
Cellular nitrogen compound & 2.33 & 0.004 \\
Regulation of nucleobase-containing & 2.33 & 0.004 \\
compound & & \\
Regulation of nitrogen compound & 0.52 & 0.005 \\
Nitrogen compound & 2.24 & 0.009 \\
Single organismal cell-cell adhesion & 2.03 & 0.013 \\
Nucleic acid & 1.86 & 0.014 \\
\hline
\end{tabular}

$\mathrm{BP}$, biological process.

Table IV. Top ten gene ontology CC terms that upregulated genes were enriched in.

\begin{tabular}{lcr}
\hline CC term & $\begin{array}{c}\text { Enrichment } \\
\text { score }\end{array}$ & P-value \\
\hline Organelle & 4.48 & $<0.001$ \\
Membrane-bounded organelle & 4.24 & $<0.001$ \\
Intracellular membrane-bounded & 3.68 & $<0.001$ \\
organelle & & \\
Intracellular organelle & 3.46 & $<0.001$ \\
SAGA-type complex & 2.97 & 0.001 \\
Nucleus & 2.83 & 0.001 \\
Nuclear part & 2.18 & 0.007 \\
Nuclear lumen & 2.16 & 0.007 \\
Histone acetyltransferase complex & 2.12 & 0.008 \\
Intracellular organelle part & 2.11 & 0.008 \\
\hline
\end{tabular}

CC, cellular component.

demonstrated that cellular localization was the most enriched biological process of DE genes, and the major cellular components involved were the cytoplasm and cytosol. The molecular function was predominantly enriched in kinase, enzyme and protein kinase binding.

KEGG analysis of DE genes. KEGG enrichment was identified for downregulated DE genes; however, not for upregulated DE genes. The top 10 most significant enrichment KEGG terms are listed in Table IX. The major KEGG item was the insulin signaling pathway, with $10.3 \%$ of downregulated DE genes involved in it (Fig. 2; http://www.genome.jp/kegg-bin/show_ pathway scale $=1.0 \&$ query $=\&$ map $=$ hsa $04910 \&$ scale $=0.67 \&$ auto_image $=\&$ show_description $=$ hide $\&$ multi_query $=$ and http://www.kegg.jp/kegg/legal.html).
Table V. Top ten MF terms that upregulated genes were enriched in.

\begin{tabular}{lcc}
\hline MF term & $\begin{array}{c}\text { Enrichment } \\
\text { score }\end{array}$ & P-value \\
\hline Histone acetyltransferase activity & 2.46 & 0.003 \\
Acetyl-CoA:L-lysine & 2.46 & 0.003 \\
N6-acetyltransferase & & \\
Ion channel activity & 2.46 & 0.003 \\
Substrate-specific channel activity & 2.42 & 0.004 \\
Channel activity & 2.34 & 0.005 \\
Passive transmembrane & 2.34 & 0.005 \\
transporter activity & & \\
Enzyme activator activity & 2.29 & 0.005 \\
Anion channel activity & 2.12 & 0.008 \\
N-acetyltransferase activity & 2.11 & 0.008 \\
GTPase activator activity & 2.09 & 0.008 \\
\hline
\end{tabular}

MF, molecular function.

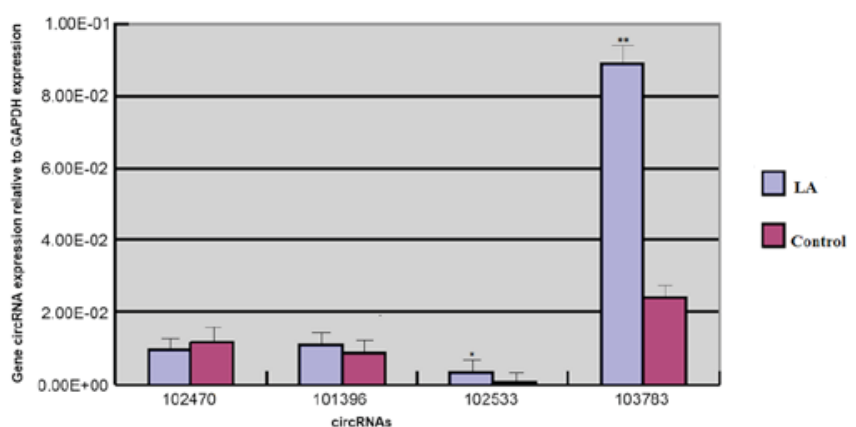

Figure 1. Mean relative expression of 4 circRNAs in LA and control groups. Mean relative expression of hsa_circ_102470, hsa_circ_101396, hsa_circ_102533 and hsa_circ_103783 was determined in LA and control groups. circRNAs, circular RNAs; LA, leukoaraiosis. ${ }^{*} \mathrm{P}<0.05$ and ${ }^{* * *} \mathrm{P}<0.001$

\section{Discussion}

At present, understanding of the pathogenesis of LA remains to be fully elucidated, and there no reliable indicator for the early diagnosis of LA in the hypertension population. Previous studies have eported that LA may be associated with various factors, including age, sex (7), hypertension (2), small vessel disease and atherosclerosis (8). Hypertension is one of the most important established risk factors for LA (9), and pulse pressure was previously demonstrated to be independently associated with LA, regardless of classical cardiovascular risk factors in elderly men (10). However, patients with hypertension do not necessarily suffer from LA. To predict the potential for development of LA among patients with hypertension, the present study investigated potential predictors at the circRNA level.

Previously, circRNA was demonstrated to be a highly prevalent RNA species in the human transcriptome (6), and they may have important roles in the regulation of gene expression (11). circRNA expression levels can be $>10$-fold 
Table VI. Top ten BP terms that downregulated genes were enriched in.

\begin{tabular}{lcc}
\hline & $\begin{array}{c}\text { Enrichment } \\
\text { score }\end{array}$ & P-value \\
\hline Cellular localization & 4.97 & $<0.001$ \\
Establishment of localization in cell & 4.92 & $<0.001$ \\
Intracellular transport & 4.39 & $<0.001$ \\
Macromolecular complex subunit & 4.26 & $<0.001$ \\
organization & & \\
Cytoplasmic transport & 4.18 & $<0.001$ \\
Modification-dependent & 4.10 & $<0.001$ \\
macromolecule catabolic process & & \\
Organic substance catabolic process & 3.98 & $<0.001$ \\
Catabolic process & 3.97 & $<0.001$ \\
Cytoskeleton organization & 3.84 & $<0.001$ \\
Organelle localization & 3.83 & $<0.001$ \\
\hline
\end{tabular}

$\mathrm{BP}$, biological process.

Table VII. Top ten CC terms that downregulated genes were enriched in.

\begin{tabular}{lcc}
\hline CC term & $\begin{array}{c}\text { Enrichment } \\
\text { score }\end{array}$ & P-value \\
\hline Cytoplasm & 8.17 & $<0.001$ \\
Cytosol & 8.16 & $<0.001$ \\
Membrane-bounded organelle & 6.11 & $<0.001$ \\
Cytoplasmic part & 5.46 & $<0.001$ \\
Intracellular part & 5.42 & $<0.001$ \\
Organelle & 5.33 & $<0.001$ \\
Intracellular & 5.12 & $<0.001$ \\
Intracellular organelle & 4.20 & $<0.001$ \\
Intracellular membrane-bounded & 4.18 & $<0.001$ \\
organelle & & \\
Macromolecular complex & 3.36 & $<0.001$ \\
\hline CC, cellular component. & & \\
\hline
\end{tabular}

higher compared with their linear isomers $(6,12)$. The two most important properties of circRNAs are highly conserved sequences and have a high degree of stability in mammalian cells (13). Certain circRNAs regulate gene expression by serving as competing endogenous RNAs (14). circRNAs block the inhibitory effect of microRNAs (miRNAs) on the target RNA by combining with miRNAs, so as to regulate the expression level of the target RNA (15). Currently, the circRNA with the most compelling evidence for a biological function is the miRNA-7 sponge, CDRlas (16). miRNA-7 was previously demonstrated to serve a key role in Parkinson's and Alzheimer's diseases $(17,18)$.

Although an increasing number of studies have investigated the potential functions of circRNAs in the brain, to the best of
Table VIII. Top ten MF terms that downregulated genes were enriched in.

\begin{tabular}{lcr}
\hline MF term & $\begin{array}{c}\text { Enrichment } \\
\text { score }\end{array}$ & P-value \\
\hline Kinase binding & 6.17 & $<0.001$ \\
Enzyme binding & 5.05 & $<0.001$ \\
Protein kinase binding & 4.53 & $<0.001$ \\
Protein binding & 3.74 & $<0.001$ \\
Transferase activity & 3.48 & $<0.001$ \\
Nucleotide binding & 3.01 & $<0.001$ \\
Nucleoside phosphate binding & 3.01 & $<0.001$ \\
Protein homodimerization activity & 2.89 & 0.001 \\
ATP binding & 2.82 & 0.002 \\
Protein domain specific binding & 2.81 & 0.002 \\
\hline
\end{tabular}

MF, molecular function.

Table IX. Top ten KEGG pathways that downregulated genes were enriched in.

\begin{tabular}{|c|c|c|}
\hline KEGG pathway & $\begin{array}{l}\text { Enrichment } \\
\text { score }\end{array}$ & P-value \\
\hline $\begin{array}{l}\text { Insulin signaling pathway, } \\
\text { Homo sapiens }\end{array}$ & 2.97 & 0.001 \\
\hline B cell receptor signaling & 2.52 & 0.003 \\
\hline Thyroid hormone signaling & 2.51 & 0.003 \\
\hline Axon guidance & 2.38 & 0.004 \\
\hline mTOR signaling & 1.86 & 0.014 \\
\hline $\begin{array}{l}\text { Transcriptional misregulation } \\
\text { in cancer }\end{array}$ & 1.77 & 0.017 \\
\hline Chemokine signaling & 1.68 & 0.02 \\
\hline $\begin{array}{l}\text { Glyoxylate and dicarboxylate } \\
\text { metabolism }\end{array}$ & 1.64 & 0.02 \\
\hline Propanoate metabolism & 1.64 & 0.02 \\
\hline Platelet activation & 1.62 & 0.02 \\
\hline
\end{tabular}

KEGG, Kyoto Encyclopedia of Genes and Genomes.

our knowledge, the present study is the first to investigate the association between circRNAs and LA. The current study was the first to construct a spectrum of differentially expressed circRNAs in patients with LA, and to identify potential biomarkers for the diagnosis of LA and its pathogenesis. At present, there is no circRNA database for LA. The present study aimed to search a circRNA database for patients with LA and hypertension, via an Arraystar Human circRNA Microarray analysis of 6 test samples and 6 control samples. Subsequently, 32 upregulated and 132 downregulated circRNAs were screened out. Furthermore, 3 upregulated circRNAs (hsa circ_101396, hsa_circ_102533 and hsa_circ_103783) and 1 downregulated circRNA (hsa_circ_102470) were selected based on a $>1.5$ fold-change $(\mathrm{P}<0.05)$, which were further 
INSULIN SIGNALING PATHWAY

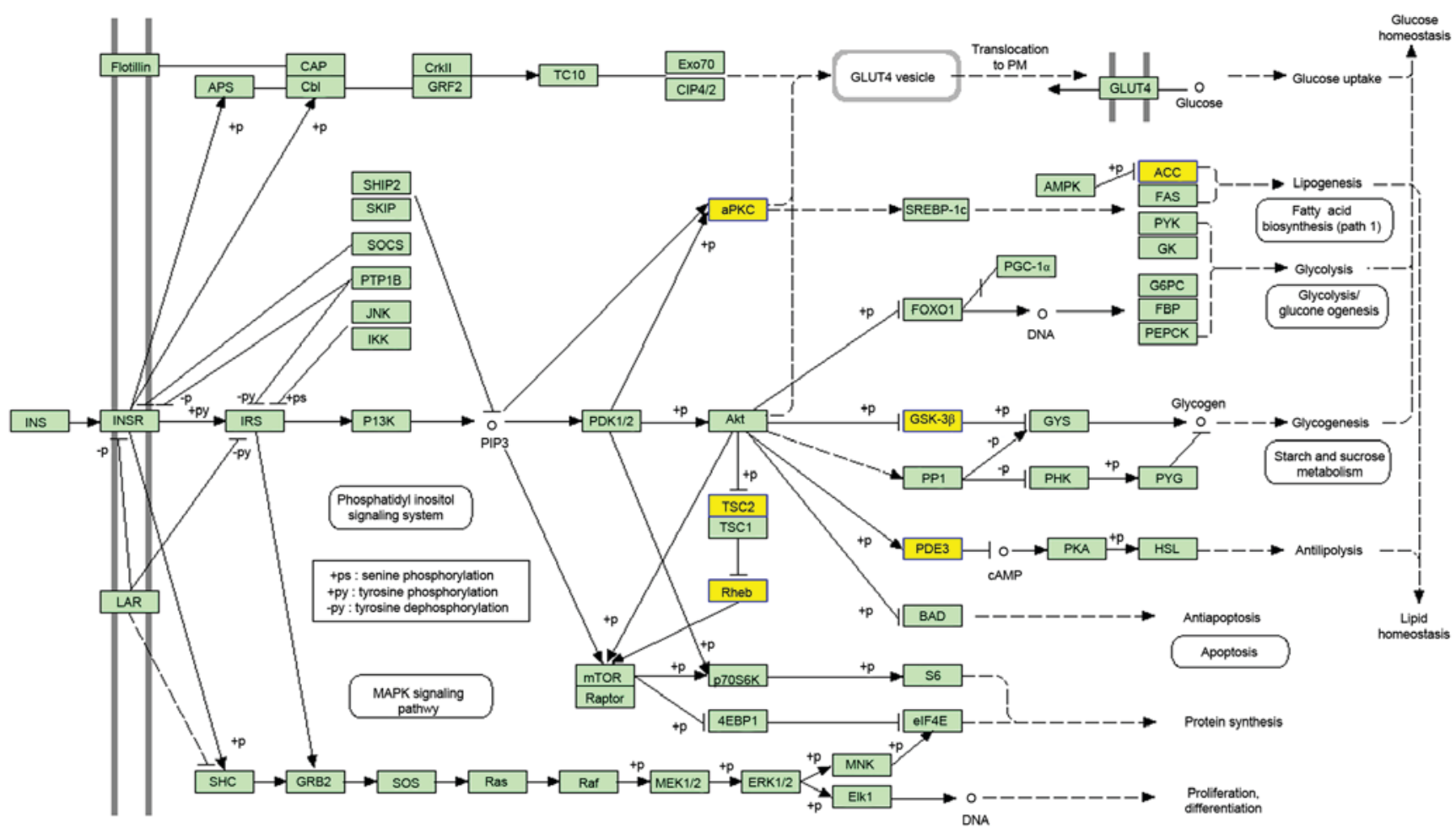

Figure 2. Insulin signaling pathway. KEGG enrichment analysis identified that genes that were significantly downregulated in patients with leukoaraiosis compared with controls were enriched in the insulin signaling pathway. The yellow boxes indicate the main compounds in the insulin signaling pathway.

assessed by RT-qPCR. The results of the current study demonstrated that the exonic RNAs, including hsa_circ_102533 (3.52-fold; $\mathrm{P}<0.05$ ) and hsa_circ_103783 (3.67-fold; $\mathrm{P}<0.001)$, were significantly upregulated in the LA patients compared with the control group, indicating the potential diagnostic value of certain circRNAs for LA.

GO enrichment analysis of the upregulated DE genes indicated that DE genes were primarily associated with metabolic processes, organelles and the activity of certain transferases. However, it remains to be elucidated whether such gene products have the potential to be novel detectors for LA. In addition, GO and KEGG analysis of downregulated DE genes indicated that DE genes were primarily enriched in the cytoplasm, kinase binding and the insulin signaling pathway. The insulin signaling pathway was revealed to be pleiotropic, and included c-Cbl-associated protein, insulin receptor substrate-1, mitogen activated protein kinase, plasma cell glycoprotein-1, phosphoinositide-dependent kinase, protein kinase $\mathrm{C}$ and phosphatidylinositol 3-kinase (PI3-K). The involvement of phosphatidylinositol 3-kinase/protein kinase B (PI3-K/Akt) -dependent signaling pathways were found in normal cellular functions. The dysfunction of this signaling pathway has a pivotal role in atherosclerosis (19) and hypertension (20). Therefore, it was hypothesized that the PI3-K/Akt-dependent signaling pathway may contribute to the pathological changes of LA. However, this requires further investigation.

In conclusion, DE circRNAs were identified between patients with LA and controls among a hypertension population. The present study indicated that the upregulated circRNAs, hsa_circ_102533 and hsa_circ_103783, may have potential as biomarkers for the diagnosis of LA. GO and KEGG enrichment analyses of DE genes indicated that the DE genes were associated with the pathogenesis of LA. However, due to the limited number of plasma samples of LA, the present study only analyzed the expression of 4 circRNAs in 20 plasma samples with LA and 10 control samples. Future studies should increase the sample size to further confirm the diagnostic value of hsa_circ_102533 and hsa_circ_103783 for LA. Importantly, the conclusions were based on a large number of early experimental results; therefore, the specific mechanisms requires further investigation.

\section{Acknowledgements}

This study was supported by the National Natural Science Foundation of China (grant no. 30970991) and the Key Research and Development Program of Shandong Province (grant no. 2015GSF118069).

\section{References}

1. Lin Q, Huang WQ and Tzeng CM: Genetic associations of leukoaraiosis indicate pathophysiological mechanisms in white matter lesions etiology. Rev Neurosci 26: 343-358, 2015.

2. Ben-Assayag E, Mijajlovic M, Shenhar-Tsarfaty S, Bova I, Shopin L and Bornstein NM: Leukoaraiosis is a chronic atherosclerotic disease. ScientificWorldJournal 2012: 532141, 2012.

3. Memczak S, Jens M, Elefsinioti A, Torti F, Krueger J, Rybak A, Maier L, Mackowiak SD, Gregersen LH, Munschauer M, et al: Circular RNAs are a large class of animal RNAs with regulatory potency. Nature 495: 333-338, 2013. 
4. Chen YT, Rettig WJ, Yenamandra AK, Kozak CA, Chaganti RS, Posner JB and Old LJ: Cerebellar degeneration-related antigen: A highly conserved neuroectodermal marker mapped to chromosomes X in human and mouse. Proc Natl Acad Sci USA 87: 3077-3081, 1990

5. Livak KJ and Schmittgen TD: Analysis of relative gene expression data using real-time quantitative PCR and the 2 (-Delta Delta C(T)) method. Methods 25: 402-408, 2001.

6. Salzman J, Gawad C, Wang PL, Lacayo N and Brown PO: Circular RNAs are the predominant transcript isoform from hundreds of human genes in diverse cell types. PLoS One 7: e30733, 2012.

7. Simoni M, Li L, Paul NL, Gruter BE, Schulz UG, Küker W and Rothwell PM: Age- and sex-specific rates of leukoaraiosis in TIA and stroke patients: Population-based study. Neurology 79: $1215-1222,2012$

8. Uh J, Yezhuvath U, Cheng Y and Lu H: In vivo vascular hallmarks of diffuse leukoaraiosis. J Magn Reson Imaging 32: 184-190, 2010

9. Avet J, Pichot V, Barthélémy JC, Laurent B, Garcin A, Roche F and Celle S: Leukoaraiosis and ambulatory blood pressure load in a healthy elderly cohort study: The PROOF study. Int J Cardiol 172: 59-63, 2014.

10. Kim SH, Shim JY, Lee HR, Na HY and Lee YJ: The relationship between pulse pressure and leukoaraiosis in the elderly. Arch Gerontol Geriatr 54: 206-209, 2012.

11. Guo JU, Agarwal V, Guo H and Bartel DP: Expanded identification and characterization of mammalian circular RNAs. Genome Biol 15: 409, 2014.
12. Jeck WR, Sorrentino JA, Wang K, Slevin MK, Burd CE, Liu J, Marzluff WF and Sharpless NE: Circular RNAs are abundant, conserved, and associated with ALU repeats. RNA 19: 141-157, 2013.

13. Hansen TB, Jensen TI, Clausen BH, Bramsen JB, Finsen B, Damgaard CK and Kjems J: Natural RNA circles function as efficient microRNA sponges. Nature 495: 384-388, 2013.

14. Danan M,Schwartz S,EdelheitS and Sorek R: Transcriptome-wide discovery of circular RNAs in Archaea. Nucleic Acids Res 40: 3131-3142, 2012.

15. Valdmanis PN and Kay MA: The expanding repertoire of circular RNAs. Mol Ther 21: 1112-1114, 2013.

16. Hentze MW and Preiss T: Circular RNAs: Splicing's enigma variations. EMBO J 32: 923-925, 2013.

17. Junn E, Lee KW, Jeong BS, Chan TW, Im JY and Mouradian MM Repression of alpha-synuclein expression and toxicity by microRNA-7. Proc Natl Acad Sci USA 106: 13052-13057, 2009.

18. Lukiw WJ: Circular RNA (circRNA) in Alzheimer's disease (AD). Front Genet 4: 307, 2013.

19. Namgaladze D and Brüne B: Phospholipase A2-modified low-density lipoprotein activates the phosphatidylinositol 3-kinase-Akt pathway and increases cell survival in monocytic cells. Arterioscler Thromb Vasc Biol 26: 2510-2516, 2006.

20. Northcott CA, Hayflick JS and Watts SW: PI3-kinase upregulation and involvement in spontaneous tone in arteries from DOCA-salt rats: Is p110delta the culprit? Hypertension 43: 885-890, 2004. 